\title{
SOBRE LOS VERBOS UMBÍlICOS DE LA SEMÁNTICA TOPOLÓGICA
}

\author{
Fernando Miguel Pérez Herranz \& Antonio José López Cruces \\ Universidad de Alicante \\ Perez.Herranz@)ua.es
}

A Jean Petitot y a Wolfgang Wildgen, que nos trazaron el camino de una estupenda aventura intelectual

\begin{abstract}
Resumen
In this paper we propose a verbs classification from the Topological Semantic. A distinction is made between semiophysic verbs, mainly linked to (world behaviors» and non-semiophysic verbs, which are generally metalinguistic. Among the semiophysic verbs, we essentially deal with parabolic, elliptic and hyperbolic-type verbs.
\end{abstract}

«Mas el nervio del estilo es la intensión del verbo (...) Preñado ha de ser el verbo, no hinchado; que signifque, no resuene; verbos con fondo, donde se engolfe la atención (...) Elige un verbo entre mil Cornelio Tácito, no se contenta con qualquiera Valerio, y con los muchos borrones iluminaron Virgilio y Marcial sus escritos. Llamo intensión la del verbo porque ay grados de propiedad en el significar; exageran unos; al contrario, otros cseasamente apuntan; y hase de exprimir a la ocasión» [B. Gracián, Arte de ingenio. Tratado de la Agudeza, Discurso XLVIII].

\section{MoRfodinÁmiCA Y SEMÁNTICA TOPOLÓGICA}

La hipótesis de la que partimos afirma que una determinada concepción dcl lenguaje no es previa a la teoría lingüística, sino una consecuencia de la propia teoría junto a postulados tomados de otras ciencias - neurobiología, psicología, antropología...-. Se rechazarán, en consecucncia, las teorías puras del lenguaje, tanto las atomistas (positivistas) —el lenguaje es el resultado de unir elementos discretos (fonemas, sílabas...) - como las holistas (románticas) -no hay lenguaje, sino lenguas, unidades privilegiadas de tipo metafísico, incluso teológico (lenguas del paraíso...) - Nuestra concepción se sitúa dentro del conjunto de teorías que entienden el lenguaje como un fenómeno natural, morfológico y dinámico, y trata de explicar la correspondencia entre las estructuras lingüísticas y el proceso espacio-temporal significado. La aporía central a la que nos enfrentamos toma csta formulación: ¿Cómo es que los procesos morfológicos espacio-temporales son continuos y, en cambio, la expresión lingüística es discreta y el discurso se presenta troceado en frases nucleares? 
Frente a los modelos lingüísticos más tradicionales - regidos por la lógica, la hermenéutica, el pragmatismo o la generatividad - , los modelos morfodinámicos apelan a principios y reglas estudiados por la Topologia, cuya aplicación al lenguaje es una decisión genial del matemático y filósofo René Thom, que con su Teoria de las Catástrofes - que nosotros venimos denominando, creemos que de manera más apropiada, Teoria de las singularidades topológicas (TSS) - , ha fertilizado tanto la ciencia como la filosofia, de la misma manera en que lo hicieron Aristóteles con su teoría del silogismo o Bertrand Russcll con la Lógica Formal. Thom ha escrito que el mérito de la ciencia reside en la manera en que reduce la arbitrariedad y lo indefinido de la descripción. En este sentido, la TSS muestra cómo ciertos sistemas dinámicos inestables se pueden estabilizar mediante pequeñas deformaciones controladas por determinados parámetros. A los teoremas de Poincaré-Benedixon, que clasifican los atractores en cíclicos, toros, fuentes, sillas..., y al de Lorenz, que clasifica los atractores extraños de la teoría del Caos, Thom añadió un teorema de clasificación de singularidades en sistemas de una o dos variables y de hasta cuatro parámetros, obteniendo las ya clásicas singularidades topológicas elementales: cuatro cuspoides: pliegue, cúspide, cola de milano y mariposa; y tres umbílicas: parabólica, hiperbólica y eliptica (Thom, 1972).

Utilizando esta metodología morfodinámica, nos hemos ocupado en diversos trabajos de cuestiones relacionadas con la Semántica a la que hcmos dado el calificativo de topológica (ST), a fin de eludir las connotaciones de la palabra «catástrofe» [cf. la bibliografía de Pérez Herranz y López Cruces]. Las formas significantes están implícitas en el mundo y el lenguaje apunta hacia la significación, que no es un hecho autónomo, ya que el mundo mismo (o alguna de sus partes) cs pregnante para los organismos, pues suscita un cambio de estado visible, un efecto figurativo, en aquella entidad a la que inviste. Según csta tesis, no puede defonderse que los significados pertenezcan al «alma», al «ego» (las ideas innatas del racionalismo); pero tampoco que los significados sean meras representaciones impresas sobre una mente pasiva quam tabula rasa (las percepciones del empirismo). Los significados están constreñidos por las formas pregnantes, ${ }^{1}$ por lo que la significación característica de la frase nuclear es el reflejo de las grandes pregnancias biológicas (sexualidad, hambre, miedo...), ampliadas después por las estructuras sociales, económicas, culturales... La teoría de la saliencia y la pregnancia es el fundamento de la teoría de la inteligibilidad de Thom.

Pues bien, el matiz que hemos querido incorporar al proyecto biolingüistico de ThomPetitot-Wildgen - lo que consideramos la originalidad de nuestro trabajo- ha consistido en el establccimiento de la relación entre la Lógica y la Topología, en mostrar que la Lógica es una parte (una proyección) de la Topología y que, por tanto, ésta se convierte en el novum organum para la filosofía del siglo XXI y en deseable guía de la lingüística, que ha venido siendo guiada por la lógica durante todo el siglo XX. Los análisis lógicos permanecen limitados a las propiedades unilineales (álgebra de Boole) que proyectan las estructuras $n$-dimensionales (cf. Pérez Herranz, 1996: cap. 3).

En cualquier caso, la teoría de las singularidades topológicas de Thom ni es toda la Topología ni puede dar cuenta de todo el fenómeno lingüístico. Quede bien entendido desde

1 Por eso hay filosofias que acogen de manera natural la TSS y otras que no. Así, las filosofias fenomenológicas lo hacen por mediación de la idea de «intencionalidad» (véase Petitot) y las materialistas por mediación de la idea de «morfología» (véase Pérez Herranz). Más dificultad encuentran las filosofías positivistas, empiristas o analíticas (véase Bunge). 
el principio: la TSS habrá de acompañarse de manera orgánica de una teoría topológica más amplia que incorpore los atractores extraños, las estructuras fractales, los sistemas disipativos y sinergéticos, etc. (cf. Boutot, 1993): una ciencia que se encucntra cn sus inicios. Aunque contamos con grandes topólogos como Arnold, éstos son aún reticentes con las aplicaciones de la topologia en las ciencias no estrictamente físicas. Se requiere otra sensibilidad científico-humanista como la de Thom para proseguir su proyecto. En consecuencia, hay zonas del lenguaje que se escapan a la formalización topologica desarrollada hasta ahora (sería de gran importancia para la teoría el que se confirmase la hipótesis de Freeman (1991) de que el ccrebro se comporta como un sistema caótico).

Las lenguas particulares (inglés, castellano, chino...) pertenecen a la clase de los términos operados por seres humanos; son herramientas ( $\mathrm{E} 1$ nombre es un cierto instrumento» dice Platón en El Crátilo 388a; «El lenguaje es como una caja de herramientas», Wittgenstein cn Investigaciones flosóficas, 11) que se despliegan y se desarrollan por medio de los materiales fónicos — sonidos, sólo muy tardíamente traducidos a grafemas-y que se organizan en sistemas - lenguaje familiar, cazador, agrario, artesanal... - (Postulado I). Esta teoría tiene una larga tradición: Anaxágoras advirtió ya hace veinticinco siglos que «el hombre piensa porque tienc manos»; recientemente, Frank R. Wilson ha establecido los vínculos neurofisiológicos entre la mano y el lenguaje; y Mottron ha puesto de relieve las conexiones entre el lenguaje y el contacto visual.

La primera consecuencia del primer postulado para la Lingüística es que la Semántica ha de ponerse en un estadio muy anterior no sólo a la Ciencia, sino a la misma Ontología (corolario 1). Piaget ha mostrado cómo el desarrollo cognitivo del niño pasa por un estadio en el que la representación de los objetos es más semántico que geométrico. La historia de la filosofía, por su partc, muestra, como lo defienden los escolásticos realistas, que los humanos no estamos determinados por los concretos individuales, ya que el entendimiento es capaz de conocer los universales, en los que se funda la libertad humana, pues gracias a ellos el hombre puede elegir entre sus inferiora. Y llamamos Semántica precisamente a ese conjunto de posibilidades significativas. Ahora bien, cstos núcleos con sentido, significativos, han de mostrarse públicamente. Como la constitución evolutiva de los seres humanos ha utilizado sonidos que poseen una estructura lineal, la estructura tridimensional de los fenómenos mundanos (tetra-dimensional, si incorporamos el tiempo) requiere que la significatividad se conecte de manera interna con la Sintaxis del habla, con el orden de los sonidos (el orden de los grafemas, las palabras y los sintagmas de la lengua escrita es algo muy posterior y poco relevante para la teoría morfodinámica del lenguaje). Así, nos encontramos con el problema más difícil que ha de afrontar la lingüística: la conexión entre la Semántica y la Sintaxis, problema que se ha venido planteando a lo largo del tiempo en campos muy diferentes (Cifuentes, 1994: 65-111).

La filosofia fue muy sensible desde su inicio a esta cuestión: ¿Cómo se conecta la sintaxis con la mente humana y cómo es capaz de expresar los hechos del mundo? Un problema que fue planteado, ya en el inicio mismo del pensamiento heleno, por Gorgias (Pérez Herranz, 1999). El arte también se ha visto envuelto en esta cuestión: el problema de los escorzos, de la tridimensionalidad de la figura en el lienzo bidimensional (Paolo Ucello). 
$\Lambda$ simismo en etología / antropología: la diferencia entre el hombre y el primate superior tiene que ver con la amplitud semántica del hombre respecto de la restricción ontológica del animal. Las formas pregnantes del animal son muy pocas en comparación con las de ser humano. Esta observación nos conduce a la ncurología, pues parece que este proceso tiene que ver con el aumento de la capacidad cerebral: el proceso de cerebración es un proceso de semantización a partir de núcleos pregnantes conceptuales que se enriquecen por medio de múltiples parametrizaciones. Por eso el ser humano es tan sensible a su cercbro: por una parte, su capacidad de adelantarse a la acción (anamnesis / prolepsis: recuerdos de experiencias pasadas / estrategias para el porvenir) a partir no sólo de recuerdos, sino de recategorizaciones (en terminología de Gerald Edclman), puesto que la memoria no es un mero proceso dc almacenaje, sino un proceso activo de reordenación de estructuras neuroconceptuales.

La segunda consecuencia del primer postulado es que han de existir universales semánticos para que podamos compartir información con otros seres dotados de sistema nervioso, pues los signos fónicos no son significativos por sí mismos (corolario 2). Aquí entra la gran aportación de Chomsky a la lingüística dentro de la tradición estoica (que nos excusa de añadir nada más).

Aceptamos, entonces, la continuidad estructural entre: el nivel de organización del mundo (ontología) $\leftrightarrow$ la percepción $\leftrightarrow$ la memorización (neurofisiología) $\leftrightarrow$ la estructura lingüística ${ }^{2}$ La cnunciación de un mensaje habrá de entenderse como el efecto figurativo debido a una pregnancia que calectiza (condiciona) al sujeto. Por ejemplo: surge una saliencia que excita la psique; algo que aparece y que aún no ha sido identificado, resalta en el continuo espacial: «Allí, al otro lado del río...»; se enuncia el tipo de pregnancia catectizante «...avanza...(algo que se siente como un peligro inminente)...»; por fin se reconoce la fuente de la pregnancia y ésta se conceptualiza («...un tigre»). La oración nuclear es un vector de prcgnancia, de manera que si en un flujo informativo se presenta una catástrofe exterior («El tigre ataca a un individuo del grupo»), el intelecto humano somete la situación continua a una escisión mediante una oración nuclear: el verbo «ataca» arrastra al nombre «tigre» y a los deícticos (《al otro lado del río») para localizar a los actantes. Para alcanzar la inteligibilidad, csa catástrofe exterior es esquematizada mediante una estructura topológica, que corresponde a una cuspide: dos actantes y el de potencial mayor cae sobre el otro, lo que llevará a que los dos se confundan en uno (el tigre devora a su presa). La significación de ese pensamiento es la forma del proceso subyacente (Riemann). Aristóteles ya intuyó que el acto crea estados de privación, que son saturados en la dinámica del proccso. Thom dice que el verbo salura esa privación al evocar actantes, los cuales pueden entrar a su vez en privación si son excitados. La catástrofe excita la psique y se moviliza el género (que es intersubjetivo), el espacio de interacción. Se dice que dos cualidades $a$ y $b$ pertenecen al mismo gćncro si es posible encontrar una deformación continua que transforma $A$ en $B$

2 De ahí que filosolia, antropología, psicología, neurofisiología de la percepción y álgebra / topología condluyar en la disciplina que ha tomado el nombre de ciencias cognitivas. 
(cventualmente a través de otras entidades). Así, el color es un género, pues se puede pasar, por ejemplo, del rojo al azul.

Una vez que hemos mostrado de manera sintética la conexión entre el comportamiento espacio-temporal, el cerebro y el lenguaje (Semiofisica), especificaremos la naturaleza dcl método elegido, la Semántica Topológica:

- Nos interesamos por la comprensión, por la inteligibilidad, más que por la simulación, de los procesos morfodinámicos. El lenguaje ha sido estudiado en los contextos pragmáticos y lógicos fundamentalmente. Pero los teoremas de limitación de la lógica (Gödel) echaron por tierra la esperanza de una Sintaxis autónoma; sicmpre queda el resquicio para la intuición, es decir, para la significación, para la Semántica. Y frente al carácter arbitrario del signo, se reivindica la iconicidad y la relación causal entre el significante y cl significado.

- La interpretación de las frases nucleares se lleva a cabo mediante grafos que representan (en el sentido matemático) la topología espacio-temporal del proceso descrito. Los grafos de interacción elementales corresponden a las singularidades topológicas, en una y dos variables y hasta cuatro parámetros: las llamadas cuspoides (pliegue, cúspide, cola de milano, mariposa) y umbílicas (hiperbólica, elíptica y parabólica).

- La tesis central de la Semántica Topológica sería ésta: El verbo es el portador intrínseco de la significación (Fichte, Tesnière) y organiza la estructura sintáctica según relaciones actanciales. Por tanto, los modelos morfodinámicos están en deuda con el estructuralismo, y sobre todo con Tesnière y con Greimas.

- La sintaxis del habla es uno de los modos de manifestación de esos núcleos semánticos que son los verbos, to que necesariamente nos conduce a suponer una teoría sobre la naturaleza de los organismos animales. De esta sintaxis sólo podemos saber, en principio, por medio de este regressus, que es una entidad discreta y no contimua. Por eso la sintaxis ha de movcrse en el ámbito de la costumbre y sólo será normativizada mediante una lógica (ciencia de lo discreto) cuando los procesos culturales sean muy refinados.

- La categorización es posiblc, porque los mediadores entre el espacio-tiempo y las categorías, los esquemas trascendentales de la imaginación, están dotados de un logos semántico (de una semiótica).

- Un resultado de la neurofisiología ralifica la tesis de la división semántica / sintaxis: los procesos de la adquisición de la semántica (significado de las palabras) y de la sintaxis (construcción del lenguaje) son de diferente naturaleza y están asociados a diferentes áreas del cerebro (Mora, 2001: 67).

Para aplicar la Teoría de Singularidades Topológicas al lenguaje según el postulado de Thom-Riemann, se han de establecer algunos principios de correlación entre los clementos

3 Los géneros son una especie de preprogramas, al modo de un filtro o cedazo, cuya forma está moldeada según circunstancias exteriores (el ADN). Géneros unidimensionales (parejas de contrarios...); géneros bi-dimcnsionales (punto triple...)... 
del modelo topológico y al lenguaje. Son dos y los denominaremos: Principio de correlación de Petitot y Principio de correlación de Wildgen.

Principio de correlación de Petitot. Petitot trata las trayectorias como infraestructuras topológicas, sistemas de lugares y posiciones, que definen contenidos locales a los contcnidos semánticos denotados por los símbolos, y que operan sobre aquéllos y constituyen una dimensión autónoma y primaria del sentido (Deleuze, Lógica del sentido): la negación, la conjunción y la disyunción, decimos nosotros, son propiedades lineales, lógicas, de ese semantismo. Asi, Petitot al construir el cuadrado semiótico muestra cómo la catástrofe de conflicto corrcsponde a la oposición cualitativa, la conjunción y la disyunción; la catástrofe de bifurcación, a la oposición privativa: la bifurcación da lugar a la negación, y la cúspide, al conflicto (dialéctico).

Principio de correlación de Wildgen. Wildgen distingue: a) Los atractores estables de una catástrofe elemental sc interpretan como entidades semánticas estáticas. Sus realizaciones características son sustantivos, adjetivos y términos. En lo que concierne a las estructuras cognitivas, son interpretadas como clases «naturales» de individuos, objetos, cualidades... b) Los accidentes dinámicos que se suceden a lo largo del conjunto de bifurcación son fundamentalmente verbos y expresiones verbales (en el sentido de Tesnière: «Los verbos gobiernan la oracióny). Actúan como el centro dinámico de una forma Gestalt. Las expericncias, los sucesos, las acciones son los correlatos de la TC. $c$ ) Los arquetipos semánticos que son locales y suponen, por consiguiente, el sustrato espacio-temporal.

En este trabajo queremos exponer el desarrollo de una de las ideas de la Semántica Topológica: los verbos son los núcleos fundamentales de la estructura lingüística, lo que nos ha conducido a realizar una clasificación de los verbos según este modelo ${ }^{4}$.

\section{LOS VERBOS Y SU CLASIFICACIÓN}

La tarea fundamental es, entonces, la de clasificar los verbos que sc corresponden con las distintas clases de singularidades. Al primer postulado (P1) le añadimos un segundo postulado (P2) o hipótesis cognitiva, que dice: «Los sistemas cerebrales en relación con la interface psicofísica reflejan la dinámica del medio ambiente». Este postulado implica el corolario de las proyecciones múltiples: «La teoría del origen de las estructuras sintácticas expuesta más arriba supone que esta proyección de $\mathrm{R}^{\mathrm{N}}$ en $\mathbf{R}$ se descompone en una etapa intermedia, sobre un espacio de dimensión cuatro. Es esta realización espacio-tcmporal local la que define la estructura sintáctica de la frase nuclear, que no será sino una proyección unidimensional. La geometría de esta proyección $q: \mathbf{R}^{\mathbf{4}} \rightarrow \mathbf{R}$ define, por otra parte, la tipología de la lengua) $\gg$ (Thom, 1980a: 166. Remitimos para el tratamiento matemático-topológico, que desborda ampliamente los límites de este trabajo, a Pérez Herranz, 1996).

\footnotetext{
4 Para cllo nos hemos basado en los trabajos de R. Thom, J. Petitot, W. Wildgen, B. Pottier y P. A. Brandl, entre los autores foráneos, y en E. Bernárdez y Á. López Garcia-Molins y sus respectivas escuelas, entre nosotros. Nos han sido también de utilidad, por su tratamiento de los verbos de movimiento, algunos de los trabajos de J. L. Cifuentes Honrubia.
} 
Necesitamos, por consiguiente, un tercer postulado (P3), el momento de la esquematización (Petitot), que es subyacente a una semántica de los verbos. Hay que distinguir, sin embargo, entre los verbos que transcriben comportamientos del mundo y aquellos otros que transcriben comportamientos del lenguaje. Es fácil de comprobar que la mayoría de los verbos de las diferentes lenguas son muy artificiales y han ido incorporándose a lo largo de los sucesivos procesos culturales, a veces muy sofisticados. Asi, existen vcrbos construidos por analogía con otros verbos a partir de sustantivos: «La nada nadea» podría transformarse espacio-temporalmente, por ejemplo, en «La nada desaparece» o en «La nada hacc dcsaparecery, si cs que queremos interpretar la frase y que tenga un sentido. Entonces es necesario dotarse de un concepto de exfoliación topológica, regulado por parámetros novedosos, que provendrán de los desarrollos técnico y cultural. Por ejemplo, un verbo como «negociar» procede de la negación del sustantivo otium (nec-otium), lo que exige nuevos parámetros para espacializarlo, parámetros que habría que ir a buscar, por cjemplo, en el campo semántico de «argumentar». Es éste un ejemplo de la plasticidad de nuestro cerebro.

El lenguaje escrito, además, nos confunde en gran parte, porque, según una tesis muy aguda de Olsen (1998): «Una escritura con una sintaxis proporciona, por primera vez, una modelo adecuado para el habla). (Aunque, a su vez, la grafia impone sus propias reglas a la fonética y a la semántica. En cualquier caso, el lenguaje, él mismo, es considerado en algún momento como un comportamiento del mundo, y es éste uno de los aspectos que más complican el análisis del lenguaje, que ha de rcalizarse por mediación de las lenguas realmente existentes y no por algún modelo abstracto (lógico) o natural (introspección) de la Lengua.

\subsection{Definición del verbo según la ST}

Thom define el verbo como trayectoria dinámica. En nuestra época, y por la influencia del neopositivismo, en lugar de centrarse la atención en cl contenido ontológico del verbo, se ha tendido a construir un mecanismo formal que desemboca en el álgebra, y ello ha conducido a explicitar reglas de correspondencia entre los términos y la significación de los mismos; csta correspondencia exige una mente que, a su vez, ha de seguir una regla, lo que abre el regressus ad infinitum y provoca que lo que se gana en precisión lógico-sintáctica se pierda en significación (scmántica). En una línea que hoy se incluye en el amplio espectro de las ciencias cognitivas, Thom trata de explicar la correspondencia entre la forma verbal y el proceso espacio-temporal del significado. La dificultad es grande, porque la forma verbal se apoya de entrada en las frases nucleares (sintaxis) en las que se manifiesta el discurso frente a los procesos de referencia. Pero, ¿qué es una frase nuclear? Si se caracteriza por contener un verbo, condición para que la frase posea un carácter autónomo, entonces, la completud paradigmática es un universal del lenguaje, según Thom. El verbo es portador intrínscco de significación: ésta es la clave de la ST.

En Petitot (1992: 375), siempre muy cauto en las aplicaciones concretas de la TSS, hemos aprendido a comprender las posibilidades semiofísicas de la ST, aunque el lingüista y matemático francés no se muestra demasiado optimista respecto a los análisis semánticos y prefiere concentrarse en las estructuras semio-narrativas a fin de evitar la confusión que pucda darse entre la semántica, la semántica semio-narrativa y la semántica de las teorías lingüísticas del signo. Estamos totalmente de acuerdo en que esa confusión es muy peligrosa 
y en que la aplicación mecánica que quiso haccr Zeeman de la TC (pretendiendo explicar cualquier fenómeno desde las siete singularidades de Thom) ha perjudicado la sericdad intelectual de la misma. Pero csto no impide, nos parece, que pueda estudiarse el verbo más allá de los esquemas semio-narrativos. Para ello nos basamos en la definición del verbo como trayectoria que ofrece Thom.

— En Wildgen (1994: 128) aprendimos la posibilidad de la jerarquización de los verbos, a partir del principio semántico de inclusión dinámica: Un esquema máximo, en un dominio específico, contiene topológicamente esquemas parciales que son más simples (con menor número de atractores) y son constituyentes del esquema máximo

Los modelos de la TC, en todo caso, no pueden dar cuenta de todos los verbos, sino exclusivamente de aquellos que denominamos semiofisicos o de las formas pregnantes. Los verbos considerados han de poder desplcgarse espacio-temporalmente en el sustrato adecuado. El postulado 3 nos permite establecer la esquematización lingüística de: una acción motora, la acción de un sujeto sobre un objeto y la interacción entre agentes.

\subsection{Para una clasificación de los verbos}

Así pues, ya tenemos un punto de partida para establecer una clasificación de los verbos: A) Verbos semiofisicos, vinculados esencialmente a «comportamientos del mundo». B) Verbos no semiofisicos (denominales, deadjetivales, etc). Si se encuentra la manera de espacializarlos en un sustrato, se pueden tratar como verbos del apartado A).

B1) Verbos modales: deber, poder...

B2) Verbos que se remiten a una interconexión de verbos más simples. Por ejemplo: argumentar, negociar... A este tipo de verbos los denominaremos socráticos.

B3) Verbos metalingüisticos o cartesianos. Son verbos como la mayoría de los utilizados por Descartes en la Segunda de sus Meditaciones Metafisicas al responderse a su pregunta sobre por qué el hombre es una cosa que piensa: «Es una cosa que duda, que enitende, que afirma, que niega, que quiere, que no quiere, que imagina también y que siente...». Dudar, entender, afirmar... entendidos metalingüísticamente: «Pienso que he de ir a comprar el periódico». El verbo semiofísico es «comprar el pcriódico». Si se entiende el verbo pensar en su sentido semiofísico, es imposible reducirlo a ser («Pienso, luego soy»), como quería Descartes. Pensar es más bien un verbo de tipo mariposa, cuyos actantes son tres: «El que piensa», «aquello que se piensa» y «el mediador que los vincula» (Por eso los fenomenólogos hablan de acto intencional de la conciencia).

Nos ceñiremos ahora a los verbos semiofisicos: verbos cuspoides y verbos umbilicos (Véase la bibliografía de Thom, Petitot, Wildgen o Pérez Herranz). Esta división es acorde a la diferencia de estructura formal topológica. Las estructuras mundanas limitan, por un lado, con los fenómenos caóticos, cuando se acumulan los atractores y entonces el hablante es consciente de su dificultad para referirse a ciertas situaciones muy complejas, ante las cuales sucle echar mano de sustantivos como caos o lio, o de expresivas onomatopeyas. Mas, por otro lado, las estructuras mundanas limitan con cl ser mismo que ha de presuponerse en cualquier fenómeno. Así pues, los verbos se encierran entre dos extremos: Uno, lo que ya no se puede decir; y dos, el decir simplemente que alguien o algo es.

Los verbos semiofísicos se dividen en dos grandes grupos, que corresponden a estructuras de desigual complejidad: los verbos cuspoides y los verbos umbílicos. Los primeros 
se modelizan según una variable $(x)$ y hasta cuatro parámetros $(a, b, c, d)$ y no procuran ningún problema a las clasificaciones tradicionales de los verbos en relación con los esquemas actanciales. Responden a acciones universales como la aparición o desaparición súbitas, el movimiento o la locomoción de los sujetos, el control de un agente sobre un objeto, la interacción entrc dos agentes, etc. $\mathrm{El}$ hecho de que la clasificación verbal que nos sugiere la Semántica Topológica se ciña a unas pocas singularidades —independientemente de la necesidad de que su número haya de ser aumentado, como ha venido solicitando Wildgen -... creemos que ofrece la ventaja de evitar que el número de tipos de verbos crezca sin límite alguno, según los sucesivos criterios dispares (sintácticos, léxicos...) que vayan siendo agregados por el investigador (clasificaciones hay que dan hasta medio centenar de clases de verbos, algo bien alejado de la economía clasificatoria que nos aporta la Topología).

\section{LOS VERBOS SEMIOFÍSICOS UMBILICOS}

Los verbos umbílicos, que se modelizan según dos variables $(x, y)$ y hasta cuatro parámetros $(a, b, c, d)$, en condiciones de estabilidad muy complejas, corresponden a procesos y situaciones difíciles de significar de manera precisa. De ahí que dichos verbos requieran de muchos precisadores para alcanzar un significado claro y nítido. Se trata de morfologías muy ricas - arrancar, barajar, clavar, confundir, cortar, envolver, mezclar, penetrar, romper...- con un espacio de configuración tridimensional, lo que exige un tratamiento topológico muy complejo. Si añadimos a esto los complicados problemas matemáticos que todavía plantean (Callahan, 1977), es cxplicable que los lingüistas - incluso Pottier, Petitot o Wildgen, que utilizan la topología en sus investigaciones - no se decidan a abordar el estudio de los verbos umbílicos, que conllevan unos diagramas dinámicos y cinéticos complejos, y prefieran, en general, ejemplificar con las morfologías de los verbos cuspoides.

Los parámetros de las singularidades umbílicas están asociadas a fuerzas violentas o constructivas, a dinámicas centrífugas o centrípetas; a acciones seriadas con reiteración de singularidades menores, a las que incluyen como partes suyas por el principio scmántico de inclusión dinámica. Podemos imaginar el complejo cinetismo y dinamismo de los verbos umbílicos con unos versos del Romance de la guardia civil de García Lorca: «Los sables cortan las brisas / que los cascos atropellan»), donde las trayectorias violentas y continuas de los vuelos de los sables de los jinetes son sintetizadas con el verbo umbílico cortar. Los verbos umbílicos no suelen abundar en los textos, que generalmente se limitan a ser una colección de verbos cuspoides; por eso cuando aparecen suelen causar una fuerte impresión en el lector.

El grado de complejidad de ciertas acciones plantea muchos problemas a la hora de su verbalización. Por eso suele hablarse del reto quc supone para el hablante o el escritor describir las acciones que se dan de manera simultánea en el sistema dinámico que es una batalla, donde distintos ritmos compiten, chocan, se cntremezclan, como en una sinfonía de Wagner, con aproximaciones y alejamientos, con bruscas discontinuidades, con una dinámica muy rápida difícil de captar, en la que se hace complicado señalar los puntos frontera: unos atacan y otros se defienden; unos clavan o disparan y otros se parapetan o huyen.... Demasiados protagonistas y ritmos distintos, demasiadas intermitencias y transiciones de fase, demasiada violencia (movimientos no inerciales), demasiadas pautas de movimientos continuos y en distintas direcciones, de fuerzas dispares en acción, con un elevado gasto de 
cncrgía en trayectorias a menudo nada económicas (se sabe que las trayectorias cotidianas de los mamíferos suelen perseguir la economía en el gasto energético por medio de la realización de casi siempre las mismas trayectorias).

La lengua, que ha de trasformar (proyectar en nucstra tcrminología) estos procesos tan complejos en estructuras sintácticas unilineales, se encuentra con dificultades enormes ante cierto tipo de sucesos poscedores de una estructura eventiva tan simple en apariencia como el péndulo compuesto con el que se ejemplifica la teoría del Caos (Bergé et alli, 1988). Cada porción de un sistema dinámico es un grado de libertad (capaz de moverse con independencia): remolinos, turbulencias con pequeños torbellinos en su interior y éstos, a su vez, con otros torbellinos en su interior, hasta llegar a la viscosidad.

Cuando un hablante se encuentra ante pautas anómalas, ante ruptura de fronteras como las que observa en las transiciones, a veces muy rápidas, entre los estados líquido, sólido y gaseoso, en los fenómenos de mezclas o cn los de turbulencias, se da cuenta de que son fenómenos físicos muy dificiles de definir y de describir. Ante la imposibilidad de contar la riqueza de las acciones que contempla — antes de toda reducción científica-, el hablante, que se siente tentado al silencio, suele salirse por la tangente y reducir la complejidad ayudándose de verbos cuspoides más sencillos: en la batalla los que luchan ganan o pierden, viven o mueren...

\subsection{Los verbos umbílicos parabólicos}

El verbo parabólico es el verbo de mayor complejidad semántica (Para su estructura matemática, Gilmore, 1981). Se puede definir como una singularidad topológica que articula, dentro de un continuo, el paso de los verbos umbílicos hiperbólicos a los verbos umbílicos elípticos y viceversa. Contiene las acciones o comportamientos de ambos tipos de verbos. Así, el verbo barajar, que en algunas ocasiones hemos propuesto como un verbo parabólico-tipo, y que significa «entremezclar las cartas de la baraja para variar su orden antes de repartirlas», incluye en su semantismo:

a) Verbos de tipo elíptico como penetrar: Unas cartas penetran entre las demás del mazo.

b) Verbos de tipo hiperbólico como remover: Las cartas son removidas en sucesivos movimientos que van eliminando las puntas de las mal colocadas hasta que todas quedan integradas en un mazo sin aristas.

Barajar es cambiar de lugar, desplazar sucesivamente las cartas, de manera que sc logre con el desplazamiento de las partes un todo ordenado de distinta manera que el todo inicial. Se dan muchas singularidades reiteradas (unir + separar + unir + separar...), a través de ciertos ritmos en las acciones. Este sentido lo recogen tanto el Diccionario de uso del español de María Moliner — «1. Desordenar las cartas de la baraja separando el conjunto en dos partes y volviendo a juntarlas de modo que entren las de uno de los montones entre las de otro. 3. Por extensión, mezclar o desordenar algo como documentos a cartas»- como el Diccionario de la Real Academia: «1. En el juego de naipes, mezclarlos unos con otros antes de repartirlos. 3. Mezclar y revolver unas personas o cosas con otras»- (Destacamos con la cursiva en estas definiciones los verbos desordenar, mezclar y revolver, de tipo parabólico, que suelen ser los preferidos por los autores de diccionarios a la hora de definir los restantes verbos parabólicos.) 
Otro verbo parabólico-tipo es confindir, con el significado de «borrar o hacer desapareccr los límites o perfiles de las cosas, de modo que no se ve su separación: «La niebla confunde los perfiles de las montañas». Confundir incluye en su semantismo:

a) Verbos de tipo elíptico como adentrarse: la niebla se va adentrando por entre las montañas.

b) Verbos de tipo hiperbólico como recubrir: el paisaje va quedando recubierto por un mismo manto que impide distinguir lo distinto, las partes con sus límites respectivos, límites que han quedado difuminados, reblandecidos, removidos.

El Diccionario de la Real Academia recoge este sentido doble: «Mezclar, fundir cosas diversas de mancra que no puedan reconocerse o distinguirse. La oscuridad confunde los contornos de las cosas. 2. Perturbar, desordenar las cosas o los ánimosis.

Otros verbos que muestran este pasar de la situación elíptica a la hiperbólica y viceversa son: cavar, cuya trayectoria conjuga el sentido elíptico de picar, penetrar, clavar o hendir y el sentido hipcrbólico de remover la tierra con el pico, la pala, etc.; cortar, cuya trayectoria conjuga el sentido elíptico del penetrar y el hiperbólico de la acción de ir escarbando el instrumento cortante a fin de separar una parte de otra dentro de un todo...

Desde el punto de vista topológico, pertenecen a la clase de los verbos parabólicos - lanto integradores y constructivos como disgregadores y destructivos (sus inversos)- verbos que hemos caracterizado en otros trabajos mediante el criterio de la rclación entre los todos y las partes que los integran: arrancar, cavar, comparar, confundir, construir, cortar, decapitar, dividir, embarullar, engañar, enredar, entremezclar, escindir, estructurar, fraccionar, fundir, guillotinar, horadar, integrar, mezclar, ordenar, organizar, pulverizar, quebrar, quebrantar, revolver, romper, segar, subvertir, trastomar, trocear, turbar... Muchos de estos verbos incluyen cl prefijo «des-»: desbaratar, desbarajustar, desconcertar, descuartizar, desintegrar, desmembrar, desmenuzar, desordenar, desorejar, despedazar, desquiciar, destrozar, destruir, desvencijar...

\subsection{Los verbos umbilicos hiperbólicos}

¿Cuáles son los comportamientos del mundo a los que se asocian los verbos hiperbólicos? Pensamos en situaciones de relajamiento, recubrimiento, defensa o protección... Este tipo de verbos tiene que ver con la supervivencia, con la necesidad del ser vivo de no convertirse en presa. Se trata de verbos muy pregnantes, de auto y heterodefensa, de auto y heteroprotección. Veamos unos cjemplos, en los que utilizaremos la terminología de P. A. Brandt en su interesante artículo «Force and Form. On protecting》, incluido cn Morphologies of meaning (1995): El conejo (volumen $x$ ) se guarece del predador (A1: antagonista) ocultándose en la madriguera (A2: anti-antagonista); para protegerlo del frío (A1: antagonista), la madre cubre al niño ( $x$ ) con una toquilla (A2: anti-antagonista). En los verbos hiperbólicos, lo que está fuera, expuesto a unos peligros reales o potenciales, en un espacio inseguro, queda, tras los movimientos y desplazamientos oportunos, dentro de unos límites, protegido, resguardado de esos peligros (la pragmática aportará la valoración humana, subjetiva, sobre la finalidad o la calidad del proteger, del enterrar, del encapsular..). En algún artículo antcrior hemos aplicado a los verbos hiperbólicos el esquema cognitivo recipiente f contenedor o encapsulador, pero ha de quedar claro que el verbo hiperbólico debe verse de mancra dinámica: no son identificables, pues, las trayectorias que buscan y logran un 
dentro seguro (un refugio, una cárcel, una cueva...) con «contener» o «guardar» o «estar dentro de un contenedor», que son estados. Los verbos hiperbólicos implican actividades con desplazamientos y trayectorias, cambios de lugar, que habitualmente se focalizan al final del desplazamiento. En los siguientes versos del Cancionero de Unamuno se muestra esta dinámica: «Huyendo del chaparrón (A1) se han recogido las ranas $(x)$ en el lecho de la charca (A2) / (...) / qué contento de agazaparse así dentro de casa (A2) / mientras por fuera la tormenta (A1) azota / a los que a cielo descubierto pasan».

Se puede interpretar asimismo como hiperbólico cl movimiento de ruptura de las olas sobre la arena: formalmente elíptica en un principio, la ola finalmente se ablanda, redondea su volumen, para al fin recogerse sobre sí misma, como en un gesto de autoprotección (Pérez Herranz, 1996: 254), tal como queda dibujado en estos magnificos versos de «Agua con luz», del Jorge Guillén de Estudios: «Se inicia la curva de la ola, / Y aquel lomo tan grueso / Despliega su metal incandescente, / Que dura unos segundos; / Mas se curva apagándose, ya espuma». Cuando al final de La casa de Bernarda Alba de García Lorca dice: «Nos hundiremos todas en un mar de luto», Bernarda, que quiere alejar a sus hijas del qué dirán, de miradas y habladurias vistas como acciones de tipo elíptico, expresa con un verbo de tipo hiperbólico, hundirse, su deseo de mantener protegidas a sus hijas entre los muros de su casa.

P. A. Brandt, en su artículo mencionado más arriba, nos sugiere, desde la Force Dynamics de Talmy y la Teoria de las Catástrofes de Thom, un interesante modo dinámico de abordar los verbos hiperbólicos a base de escenas en las que aparecen: atractores fijos o móviles (personas o cosas que proteger, cercadas por un peligro o por antagonistas inmediatos o potenciales); antagonistas fijos o móviles, concretos o difusos y sin límites (el frío, el calor, el Tiempo, la Muerte, las enfermedades, los animales peligrosos, los vicios, las materias contaminantes, etc.); anti-antagonistas, repulsores o encapsuladores dcl antagonista, fijos o móviles, asociados o no al atractor (objetos protectores del cuerpo o de una parte del cuerpo, de la integridad de los seres vivos o de otros objctos; o bien personas que actúan como protectores, patrocinadores, guardaespaldas, escoltas, cooperantes, etc.).

En su Diccionario de uso del español, María Molincr ofrece generalmente el sentido de los verbos hiperbólicos a través de verbos como (re)cubrir, envolver, proteger o defender. La Topología nos invita a incluir dentro de la clase de los verbos hiperbólicos una serie de verbos unidos por la idea general de protección y defensa, de envoltura y (re)cubrimiento: anegar, abetunar, acoger/se, aconchar/se, acurrucar/se, agazaparse, albergar, alojar/se, apadrinar, asilar/se, ampararise, amurallar/se, arropar/se, auxiliar/se, convoyar, ayudar/se, bañar/se, calafatear; cobijar/se, cromar, cubrir/se, defender/se, embalar, embozar/se, encerrar/se, enclaustrar/se, encogerse, encovarse, encubrir, englobar, enterrar/se, envolver/se, escoltar, escudar/se, forrar, fortificar/se, guarecerse, miniar, parapetarse, patrocinar, preservar/se, protegerise, recubrir/se, reforzar/se, resguardar/se, revestir/se, salvaguardar/se, socorrer, sumergir/-se, sumir/se, tutelar, vendar/se... Puesto que las singularidades topológicas poseen dos sentidos, constructivo y destructivo, hay que incluir en la lista los verbos hiperbólicos «inversos»: descubrir/se, desnudar/se, desvestir/se, desproteger/se... Con el Objeto incorporado en la pieza léxica verbal, tendríamos verbos como: acorazar/se, amortajar/se, arropar/se, bizmar/se, broncear/se, chapar, dorar, embarrilar, embrear, encapuchar/se, enlosetar, encorsetar, enfangarse, engomar, engrudar, enjalbegar, enlatar, entoldar, jabonar/se, pavimentar, pringar/se... 


\subsection{Los verbos umbílicos elipticos}

Los comportamientos del mundo al que se asocian los verbos elípticos son las situaciones de arremetida, de embestida, de penetración, de perforación... Tienen que ver con la cara de la supervivencia, que complementa a la que suponen los verbos hiperbólicos: el ataque a otro actante utilizando la mediación de un instrumento, tanto natural (la garra...) como artificial (la lanza, la espada...). En otras ocasiones hemos ejemplificado este comportamiento con el verbo penetrar. El mundo está lleno de estas estructuras elípticas, simbolizables en el cono: los picos de las montañas, las púas, las espinas, etc. Indudablemente el mundo de los hombres ha desarrollado con profusión esta semántica en su uso de herramientas (alfileres, picos, destornilladores, pcrforadoras, taladradoras, etc.). Entonces, lo que en la naturaleza es una estructura morfológica que muestra lo puntiagudo, en el mundo humanizado queda del lado de la agresividad. Vázquez Montalbán recoge este temor instintivo del hombre ante los elementos cónicos en su novela Los mares del sur: «un cono metálico terminado en una afilada punta de muerte que crecía del suelo (...) La aguja amenazante era una escultura. Carvalho se sintió más seguron). Este instrumento o mediador puede entenderse también como mensajero indirecto: llevar algo mediante un instrumento. Como es obvio, los hombres han refinado y multiplicado enormemente este arquetipo semántico; pero lo relevante para nuestra interpretación es que seguimos presos, por así decir, de nuestro «cerebro evolutivo», acoplado a las morfologías naturales.

Hay que insistir en que son los parámetros materiales los que dan la escala real de los verbos: temperatura, calor, presión, resistencia, densidad, peso, volumen, permeabilidad mayor o menor de los materiales... Ejemplificaremos esta característica con los verbos elípticos: 10 cs igual pinchar, penetrar o atravesar un cuerpo homogéneo, macizo, continuo que uno heterogéneo, poroso, discontinuo, con agujeros, grietas, intersticios, cavidades.... Un ejemplo muy sofisticado puede aportámoslo Roentgen cuando trata de ver qué espesor de los distintos materiales podrían atravesar los rayos $\mathrm{X}$.

Como este proceso tiene muchos matices, los verbos van configurándose según las partes de la morfología general: así, el plano de control nos dará la traycctoria que corresponde al verbo. Por ejemplo, pinchar y penetrar incorporan el verbo pliegue entrar, pero no el verbo pliegue salir. Atravesar, por su parte, incorpora los verbos pliegue entrar y salir.

Las tres posiciones estándar de los verbos elípticos serían: pinchar, penetrar y atravesar, que corresponden a situaciones diferentes en el plano de control. Por ejemplo: tenemos los actantes (la mano, la aguja y el tejido pinchable, penetrable o atravesable) y las dos variables en las que se despliega el verbo: $x=$ tiempo ; $y=$ la acción. Si hacemos proyecciones o cortes topológicos (cf. López Cruces y Pérez Herranz, 1995b) con una sola variable, por ejemplo, la $y$, cntonces obtenemos distintos tipos de verbos: un verbo mariposa: para penetrar o pinchar hay que producir un desplacamiento, ir de un sitio a otro; un verbo cúspide: la aguja y el tejido han de unirse, entrar en contacto; un verbo pliegue: el objeto punzante desaparece todo o en parte en el interior de lo punzado, perforado (cn cl caso de atravesar reaparece tras haber desaparecido previamente); y finalmente el verbo que subyace a todos los verbos: ser.

Lo expuesto conlleva de manera implícita los límites semánticos de las expresiones elípticas, que no admitirán estructuras sintácticas del tipo: *Le picó de un lado a otro; *Le pinchó de costado a costado; * Le traspasó en el pecho; * Le atravesó en el pecho.... Estos 
verbos exigen un orden cuando se coordinan: «punza y traspasa», «pincha y traspasa», «pica y atraviesa», y no al revés. Además, hay que tener presente que los parámetros pueden incorporarse a la pieza léxica verbal: acuchillar es «penetrar con un cuchillo»; estoquear es «penetrar con un estoque», etc.

Se podría ensayar, entonces, con los distintos contextos en que estos verbos elípticos pueden aparecer. Veamos algunos:

\begin{tabular}{|c|l|}
\hline Parámetro: & Resultado \\
\hline $\begin{array}{c}\text { - medicina } \\
\text { corridas de loms }\end{array}$ & $\begin{array}{l}\text { entrepunzar, inyectar, jeringar, punzar, puncionar, trepanar, velicar... } \\
\text { acachetar, agarrochar, aguijar, aguijonear, apuntillar, banderillear, } \\
\text { cornear, descabellar, empitonar, esgarrochar, puyar, rejonear.. } \\
\text { acañaverar, acogotar, crucificar, empalar.. } \\
\text { alancear, acuchillar, amachetear, apuñalar, asaetecr, disparar, ensartar, } \\
\text { espolear, flechar, herir, hincar.. } \\
\text { guerra } \\
\text { sajerear, ojalar, puntear... } \\
\text { clavetear, empernar, enclavar, escarpiar, fresar, horadar, perforar, } \\
\text { taladrar, irepar... } \\
\text { arponear.. } \\
\text { barrenar... } \\
\text { penetrar... }\end{array}$ \\
pesca de la ballena \\
mineria
\end{tabular}

\section{Finai., CON PARTIDA DE A.JEDREZ}

Uno de los símbolos más sofisticados de la civilización es el ajedrez. No nos resistimos a la tentación de sugcrir cste juego como la mejor manera artificial de expresar las situaciones umbílicas que se dan en el mundo, lo que quizás haya contribuido a su éxito. Escribe Arturo Pérez Reverte en La tabla de Flandes: «y fue allí, en C2, donde la dama negra, para protegerse de la amenaza de la torre y para ganar una pieza, se comió el caballo». El ajedrez: un espacio acotado con conflicto de atractores, donde los diagramas cinćtico (movimiento de las piezas) y dinámico (las distintas fuerzas: de la dama, de la torre o del peón) se enfrentan por la supervivencia. Las piezas se protegen del potencial ataque destructivo de otras, a la vez que las atacan. Nuestra dama negra, para sobrevivir, se ve obligada a destruir al caballo.. Toda la complejidad de una batalla — con armas destructoras, desgarramientos de cuerpos, derramamientos de sangre...--, que exigiría para su descripción la aparición de verbos umbílicos, queda resumida en el ajedrez en un verbo de una sola variable, en un sencillo verbo cúspide (al cual los verbos umbílicos incluyen en su semantismo por el principio de inclusión dinámica): comer (las piezas se comen entre sí). Y es que el problema del lenguaje ordinario, como dice Thom, no es la verdad, sino la aceptabilidad semántica.

\section{REFERENCIAS BIBLIOGRÁFICAS}

Bergé, P., Pom eau, y Vidal, CH. (1988): L'Ordre dans le Chaos, Hermann, París.

Bernárdez, E. (1994): «De la lingǘstica catastrofista a la lingüística cognitiva», Revista de Filología Alemuna, 2, págs. 181-199.

Bernárdez, E. (1995): Teoría y epistemología del texto, Madrid, Cátedra. 
Boutot, A. (1993 ): L'invention des formes, Odile Jacob, París.

Brandt, P.A. (1995): Morphologies of Meaning, Aarhus, Aarhus University Press.

Brandt, P.A. (1998): «Domains and the grounding of meaning», en Estudios de Lingüistica cognitiva II, J.L. Cifuentes (ed.), Universidad de Alicante, págs. 467-478.

Callahan, J. (1977): «Singularities and Plane Maps II», American Mathematical Monthly, n 84, págs. $765-803$.

Cifuentes, J.L. (1994): Gramálica Cognitiva. Fundamentos criticos, Madrid, Eudema.

Cuenca, M. J. y Hilferty, J. (1999): Introducción a la lingüistica cognitiva, Barcelona, Ariel.

Fosgaard, L. (1998): «Las clases de palabras», Esiudios de Lingïistica cognitiva Il, J. L. Cifuentes (ed.), Universidad de Alicante, págs. 575-592.

Frceman, W. (1991): «Fisiología de la percepción», Investigación y ciencia, $n^{\circ} 175$, págs. 30-51.

Gilmore, R: (1981) Catastrophe Theory for Scientists and Engineers, Dover Publications, New York.

Lacorre, P. (1997): «Sur un nouveau typc de représentation catastrophiste pour les modélisations en biologie et sciences cognitives», Intellectica, núm. 24, págs. 109-140.

López Cruces, A. J. y Pérez Herranz, F. (1994): «Poemas «visualizados» a través de la teoría de las catástrofes de R. Thomm, Actas del Primer Congreso Internacional de Ontología, Publicacions de la Universitat Autònoma de Barcelona, págs. 543-555.

López Cruces, A. J. y Pérez Herranz, F. (1995a): «Para una formalización «topológica» de la semántica», Estudios de Linguíistica, no 10, Universidad de Alicante, págs. 218-314.

López Cruces, A. J. y Pérez Herranz, F. (1995b): «Estudio semántico-topológico de El cementerio marino de Paul Valéry: II) Análisis semiformalizado del texto», en C. Martín Vide, Aclas del XII Congrés de Llenguatges Naturals i Llenguatges Formals», PPU, Barcelona, págs. 283-298.

López Cruces, A. J. y Pćrez Herranz, F. (1998): «Estudio de la preposición desde la senántica topológicam, en Estudios de Linguíistica cognitiva II, J. L. Cifuentes (ed.), Universidad de Alicante, págs. 817-838.

López Cruces, A. J. y Pérez Herranz, F. (2001): «Topología y literatura. Algunas notas sobre Pueblo de Azoríny, U. F. R. Lettres, Langues el Sciences Humaines, Centre Universitairc de Recherche Scientifique Université de Pau et des Pays de 1'Adour, Gardonne, págs. 209-231.

López García-Molins, A. (1989): Fundamentos de gramática perceptiva, Madrid, Gredos.

López García-Molins, A.. (1996): «Teoría de catástrofes y variación lingüística», Revista Española de Linguiistica, núm. 26, 1, págs. 15-42.

López García-Molins, A. (1998): Gramática del español. III. Las partes de la oración, Arco/Libro, S.L., Madrid.

López García-Molins, A. y Morant, R. (1998): «La posición de la lingüisticá cognitiva en el desarrollo histórico de los modelos gramaticales», Estudios de Lingüistica cognitiva II, J.L. Cifuentes IIonrubia (ed.), Universidad de Alicante, págs. 319-327.

Manca, V. (1996): «Thom's logoi in metagrammatical representations», XII Congreso de Lenguajes naturales y lenguajes formales, en Carlos Martín Vide (ed.), PPU, Barcelona, 1996, págs. 237 250.

Mora, F. (2001): El reloj de la sabiduria, Alianza, Madrid.

Moreno Cabrera, J.C. (1994): Curso Universitario de Lingüística General. Tomo II: Semántica, pragmática, morfología y fonología, Síntesis, Madrid.

Ocstcrgaard, S. (1998): «Verbal coding of dynamic proceses», Estudios de Lingüística cognitiva II, J. L. Cifuentes Honrubia (cd.), Universidad de Alicante, págs. 789-803.

Olsen, D.R. (1998): El mundo sobre el papel, Gedisa, Barcelona.

Pérez Herranz, F. (1994): «La fundamentación lógica y la tcoría de las catástrofes». Actas del Primer Congreso Internacional de Ontologia, Publicacions de la Universitat Autònoma de Barcelona, págs. 291-302. 
Pérez Herranz, F. (1995): «Descripción fenomenológica y hermenéutica matemática», en C. Martín Vide (ed.), Actas del XI Congrés de Llenguatges Naturals i Llenguatges Formals», Barcelona, PPU, págs. 301-316

Pérez Herranz, F. (1996): Lenguaje e intuición espacial, Alicante, Instituto de Cultura «Juan GilAlbert».

Pérez Herranz, F. y López Cruces, A. J. (2001): «bl Cancionero de Unamuno. Aproximación desde la semántica topológica), Fstudios de lingüistica, 15, Universidad de Alicante, págs.335-360.

Petitot, J. (1992): Physique du Sens, París, Editions du CNRS.

Petitot, J. (dir). (1988): Logos et Théorie des Catastrophes, Patiño, Genève.

Pottier, B. (1992): Sémantique générale, París, P.U.F.

Pottier, B. (1993): «Pensée et cognition», Faits de langues, París, págs. 97-103.

Pottier, B. (1998): «La topología y los esquemas mentales», en Estudios de linguíistica cognitiva, II, J.L. Cifuentes Honrubia (ed.), Universidad de Alicante, págs. 857-862.

Pottier, B. (1993-95): Semantique des representations mentales (Topodynamique cognitive), Recueil d'articles.

Santos Domínguez y Espinosa Elorza (1996): Manual de Semántica histórica, Madrid, Síntesis.

Thom, R. (1980): Modèles mathématiques de morphogenèse, París, Christian Bourgois Editeur.

Thom, R. (1988): Esquisse d'une sémiophysique, París, InterÉditions.

Thom, R. (1990): Apologie du logos, París, Hachette.

Wiesengrund, S. (1994): Topograms: an Enquiry concerning Textual Ontology, Bachelor Press, Manchestcr.

Wildgen, W. (1981): Catastrophe Theoretic Semantics. An Elaboration and Application of René Thom's theory, Amsterdam, Benjamin, 1982.

Wildgen, W. (1994): Process, Image, and Meaning, John Benjamins Publishing Wildgen, W. Company, Amsterdan / Philadelphia.

Wildgen, W.. (1999): De la grammaire au discours. Une approche morphodynamique, European Semiotics / Sémiotique Européenne, vol. 1, Petcr Lang.

Wildgen, W. (2002): «Modelos de predicación y clasificación dinámica oracional de los verbos y de los papeles configuracionales», Estudios de Lingüística, 16, Universidad de Alicante, págs. 219253. 